\title{
MASSOTERAPIA CON PALLE: STORIA E TECNICA DI APPLICAZIONE
}

\section{RECENSIONE ARTICOLO}

BESSA, Vicente Alberto Lima ${ }^{1}$

BESSA, Maria Fátima de Sousa ${ }^{2}$

BESSA, Vicente Alberto Lima. BESSA, Maria Fátima de Sousa. Massoterapia con palle: storia e tecnica di applicazione. Revista Científica Multidisciplinar Núcleo do Conhecimento. anno 04, Ed. 10, Vol. 02, pp. 05-15. nell'ottobre 2019. ISSN: 24480959, Collegamento

accesso: https://www.nucleodoconhecimento.com.br/salute/massoterapia-con-palle

\section{RIEPILOGO}

La massoterapia è stata utilizzata al logo dell'esistenza dell'umanità per vari scopi e ci sono diversi tipi di massaggio che vanno dal classico al trascendentale. Tra le varie modalità di massaggio c'è massaggio con palle. Si tratta di un massaggio molto efficiente e poco indirizzato nella letteratura attuale, così il presente studio è stato condotto e volto a indagare l'origine di questo massaggio e descrivere le tecniche di applicazione. A tal fine, è stata condotta una ricerca bibliografica basata su libri e

\footnotetext{
${ }^{1}$ Maestro in Scienze della Motricità Umana; Specializzazione in Fisioterapia Dermatofunzionale; Specializzazione in Allenamento Sportivo e Trainner Personale; Specializzazione in Ginnastica Medica; Specializzazione in argomenti avanzati in fisioterapia; Specializzazione in portoghese; Specializzazione nell'insegnamento innovativo dell'istruzione superiore; Laureato in Fisioterapia; Tecnologo in Estetica e Cosmetica; Laurea e Bachelor in Educazione Fisica; Laureato in Lettere; Laureato in Pedagogia.

2 Maestro in Scienze della Motricità Umana; Specializzazione in Ginnastica Medica; Laureato in Fisioterapia; Laurea e Bachelor in Educazione Fisica.
} 
banche dati ottenuti presso Scielo e Scholar Google. Si è scoperto che questo tipo di massaggio è stato eseguito nella dinastia Ming e oltre al massaggio potrebbe essere eseguito esercizi con le palle. $\mathrm{Ci}$ sono fondamentalmente quattro tecniche di massaggio come le palle.

Parole chiave: massaggio, massaggi, massaggi come palle.

\section{INTRODUZIONE}

Una delle terapie più antiche che è stato utilizzato fino ad oggi è la massaggiatoria e ha diverse estremità come diminuzione delle tensioni, rilascio di tossine, promuovere il rilassamento muscolare, combattere l'affaticamento e stimolare la circolazione sanguigna. (CANNECCHIA, 2019).

II massaggio è una pratica comune nel corso della storia dell'umanità ed è stato ampiamente affrontato in letteratura. Oggi, ci sono diversi video di massoterapia che possono essere facilmente accessibili su internet, su Facebook, Instagram, YouTube, WhatsApp, ecc. Tuttavia, è necessario sottolineare che non tutto ciò che viene pubblicato nei media digitali è corretto e questo crea molta confusione per i laici e principianti nel mondo dell'estetica. Pertanto, è importante sviluppare studi che consentano la corretta divulgazione delle varie tecniche di mmatoterapia e dei suoi reali benefici.

È noto che ci sono diversi tipi di massoterapia, come: svedese, tailandese, hawaiano, tantrico, indiano, ecc, ma una delle tecniche meno descritte è il massaggio con le palle, o semplicemente il massaggio con le palle. È una tecnica di massaggio che viene applicata ai tessuti molli ed esercita tre effetti fondamentali: meccanici, fisiologici e psicologici. La sua applicazione segue principi simili alla massaggiaterapia svedese, tuttavia, utilizzando palle per massaggiare la regione del corpo.

È noto che c'è una mancanza di letteratura scientifica sull'uso del massaggio a sfera, quindi il presente studio è stato condotto e mirato a indagare l'origine di questo massaggio e descrivere le tecniche di applicazione. A tal fine, è stata effettuata una 
ricerca bibliografica basata su libri e banche dati ottenuti a Scielo e Scholar Google e ha avuto come descrittori: massaggio, benefici di massaggio, massaggio palle.

\section{REVISIONE DELLA LETTERATURA}

\subsection{STORIA DEL MASSAGGIO}

Ci sono documenti storici che dimostrano che anche le prime civiltà selvagge, oltre a quelle civili, hanno già praticato un certo tipo di massaggio, in particolare attrito (WOOD e BECKER, 2008). Era una pratica comune nelle antiche civiltà indiane, cinesi, giapponesi, greche e romane. È noto che uno dei riferimenti più antichi appare in Nei Ching, che è un testo medico cinese che è stato scritto in un periodo precedente al 1500 aC. Più tardi, gli scritti sono stati trovati sul massaggio da parte dei medici ippocrate nel V secolo aC. e Avicenna e Ambrose Stop, rispettivamente, nel X e XVI secolo. (CASSAR, 2001).

Tuttavia, gli egiziani hanno già applicato il massaggio a scopo terapeutico a più di 4000 a. C. E in India, il massaggio ha cercato una visione olistica per bilanciare corpo, mente e spirito ed essere una delle raccomandazioni e insegnamenti. È noto che la descrizione del massaggio si trova nei libri Ayur Veda che sono stati scritti a circa 1800 aC. Tuttavia, è noto che quasi tutte le principali culture del mondo hanno proposto in dettaglio le indicazioni e i benefici del massaggio che è stato comunemente combinato con altri tipi di trattamento tradizionale, in particolare i trattamenti da bagno. (SILVA, 2016).

È necessario evidenziare che Ippocrate (480 a.C.) chiamato massaggio di anatripsis, il cui significato è "rubare il tessuto", in seguito il termine è stato tradotto nella parola latina frictio, che significa "attrito" o "sfregamento". È noto che questo termine predominava per lungo tempo ed è stato utilizzato negli Stati Uniti fino al 1870. In India, il massaggio è stato chiamato shampoing; in Cina, Cong-Fou; in Giappone, Ambouk (CASSAR, 2001). 
A sua volta, Ippocrate (460-370 aC) ha anche usato il massaggio come trattamento, perché credeva che il corpo è in grado di auto-guarire se adeguatamente stimolato. L'Asclepàades de Bitània era un altro medico greco che usava massaggi, diete ed esercizi per il trattamento delle malattie. Inoltre, è noto sapere che le civiltà primitive australiane, egiziane, russe, ucraine, del Pacifico e del Nord e del Sud America usavano il massaggio attraverso movimenti di attrito con oli e acque come un modo per scacciare demoni e spiriti che hanno generato malattie nelle persone e quindi purificato il corpo dei malati. Pertanto, diverse personehanno usato e raccomandato massaggio a beneficio della salute degli esseri umani. (BERTOJA e TOKARS, 2018).

Tuttavia, il massaggio non è sempre stato ben considerato, con l'ascesa del cristianesimo nel Medioevo (il periodo storico che va dal V secolo al XV secolo), il culto del corpo è venuto a essere considerato un peccato. Pertanto, la cura dell'igiene non è più presa e quindi il massaggio è stato vietato nelle civiltà crestate. (BRAUN e SIMONSON, 2007). In Europa, la Chiesa cattolica considerava il massaggio un peccato e la sua associazione con le erbe medicinali era considerata un atto di stregoneria. (RAMOS, 2017).

Con la fine del Medioevo e l'emergere del Rinascimento, il massaggio è stato scoperto e applicato di nuovo e diversi autori hanno contribuito a questo fatto. Tuttavia, fu tra il 1776 e il 1839 che Pehr Henrik Ling rivoluzionò la pratica degli esercizi e del massaggio. Ha creato le terminologie e la tecnica del massaggio svedese che è anche conosciuto come massaggio classico o guarigione dal movimento svedese. La sua tecnica è stata inizialmente diffusa in Europa e Russia e successivamente globalizzata (WOOD e BECKER, 2008).

È stato anche Ippocrate a descrivere i benefici del massaggio associato alle proprietà chimiche degli oli utilizzati (FRIT, 2002). Pertanto, si può vedere che non solo il massaggio potrebbe portare benefici, ma anche la proprietà chimica di alcuni oli vegetali ed essenziali.

La parola massaggio deriva dalla massa greca che significa "impastare" che significa toccare, maneggiare, restringere (FRITE, 2002). Tuttavia, il termine massaggiatore è 
attualmente preferibile, poiché la parola terapia indica l'uso terapeutico del massaggio. Al giorno d'oggi, ci sono diverse tecniche di massaggio tra di loro: rilassante, sport, terapeutico, modellazione, massaggio rapido, riflessirico, tantrico, ecc. Proprio come sono emersi diversi tipi di attrezzature che aiutano il terapeuta ad applicare il massaggio, tra questi ci sono le palle baoding $($ a $\ldots \ldots \ldots \ldots \ldots \ldots \ldots \ldots \ldots$

Le palle di baoding (Figura 1) sono di origine cinese e la loro denominazione proviene dalla città di baoding, situata nella provincia di Hebei nella Repubblica Popolare Cinese. Sono artefatti utilizzati per esercitare le mani e fornire relax, diminuendo lo stress. Tradizionalmente, sono composti da un paio di palline di ferro di piccole dimensioni, che sono memorizzate in una piccola scatola rettangolare. Queste sfere provengono dalla dinastia Ming (1368-1644 d.C.) e sono state fatte da un artigiano delle armi guidato da un sogno celeste. Ha creato due sfere di ferro basate sul concetto di yin/yang: una che rappresenta il ruggito del drago e l'altra l'angolo della Fenice.

In un primo momento, l'imperatore della Cina è stato affascinato dai benefici terapeutici forniti dalle palle e li ha resi uno strumento che potrebbe essere utilizzato solo dalla famiglia reale. Più tardi, palle di ferro divenne popolare e ha raggiunto il mondo. Inizialmente, sono stati utilizzati per migliorare la condizione fisica e il temperamento del treno (diminuire l'ansia, calmare) scopo per il quale sono ancora utilizzati oggi. Sono un apparato per rilassare i muscoli e le articolazioni e per la difesa nella pratica delle arti marziali.

Al giorno d'oggi, le palle baoding sono fatte di più tipi di materiali, ma rimangono leggeri da trasportare. Sono più spesso vuoti e realizzati in acciaio cromato e contengono guizos all'interno. I guizos forniscono suoni che possono suonare come campane rilassanti che rispondono alla natura e all'influenza delle cinque dita, quindi esercitano i muscoli e attivano la circolazione sanguigna. 
Figura 1: Palle baoding.

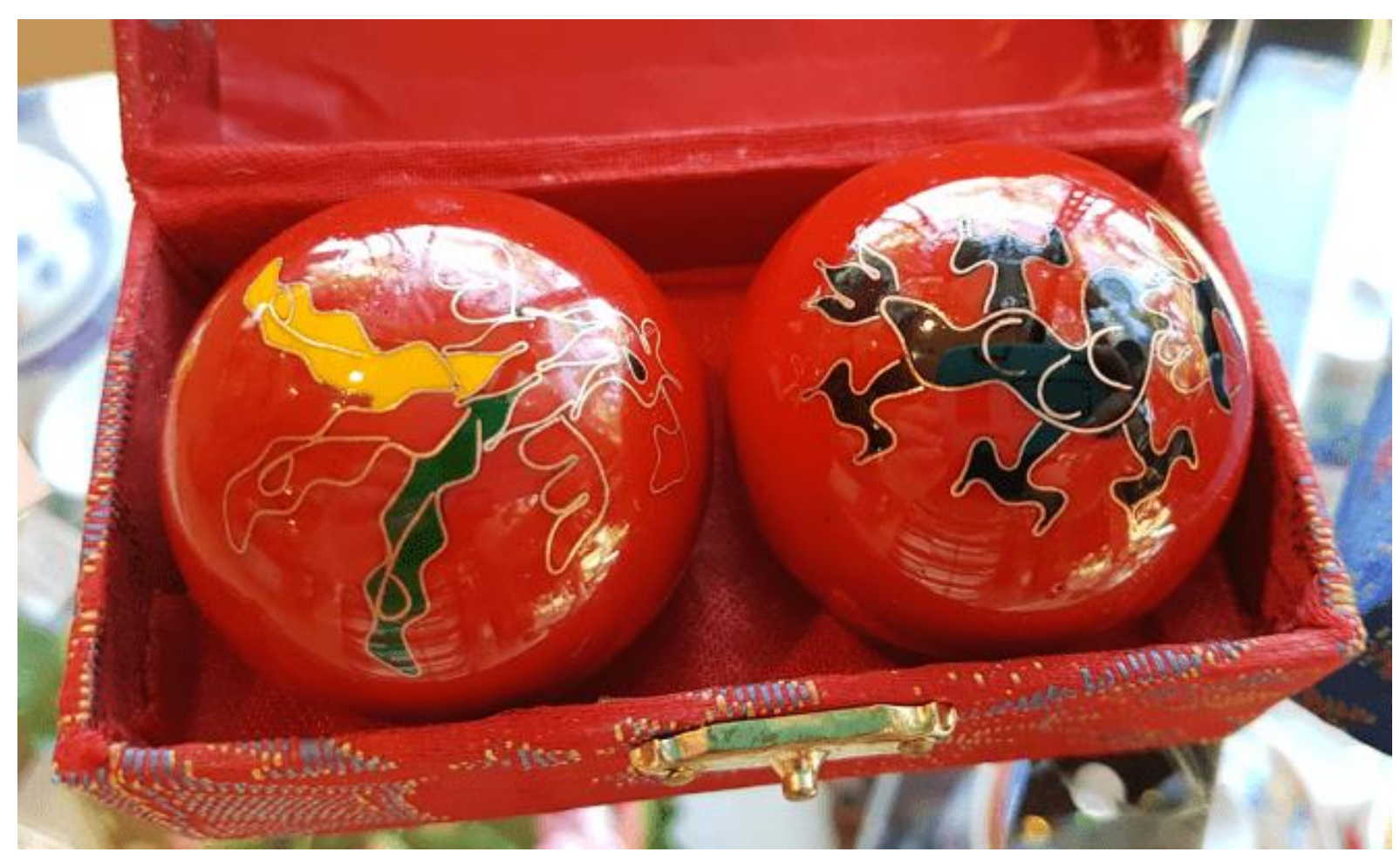

Fonte: Os autores

Con lo sviluppo di nuovi materiali e l'evoluzione della tecnica, le palle hanno cominciato ad essere prodotte con materiali magnetici, legno, silicone e metallo, gomma, silicone ecc. (Figura 2)

Figura 2: Diversi tipi di palle.
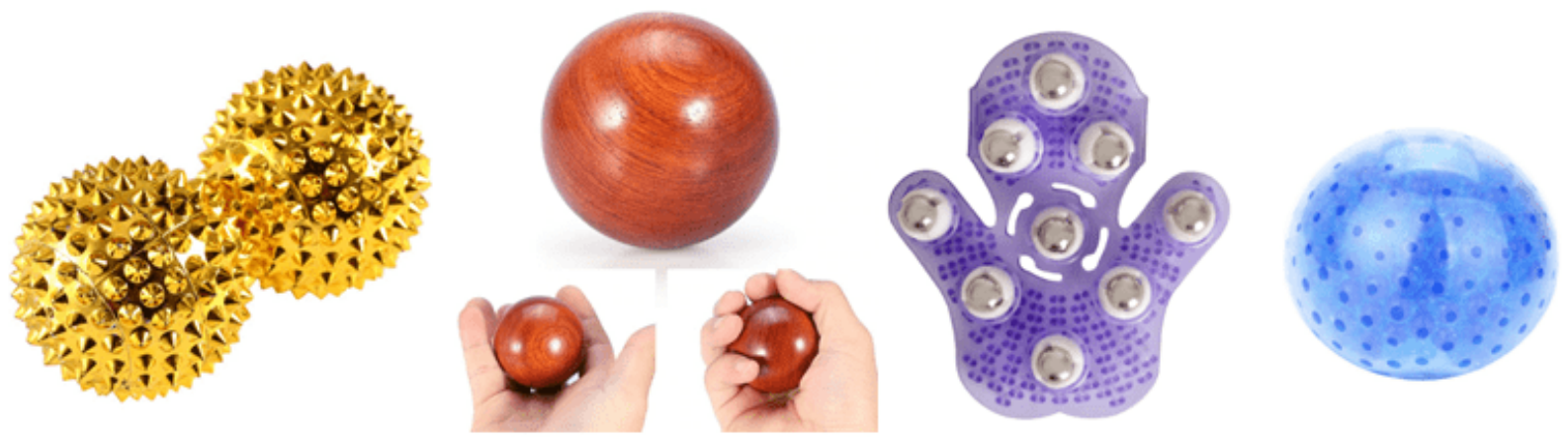

Fonte: Os autores 
Quando apparvero le sfere di ferro, erano uniche a massaggiare le mani. La persona si auto-massaggiato girando le palle tra le dita. Questa tecnica è ancora ampiamente utilizzata, ma è possibile massaggiare qualsiasi parte del corpo con l'aiuto del terapeuta o anche di varie parti del corpo attraverso l'auto-massaggio. (Figura 3)

Figura 3: Auto-massaggio con palla.
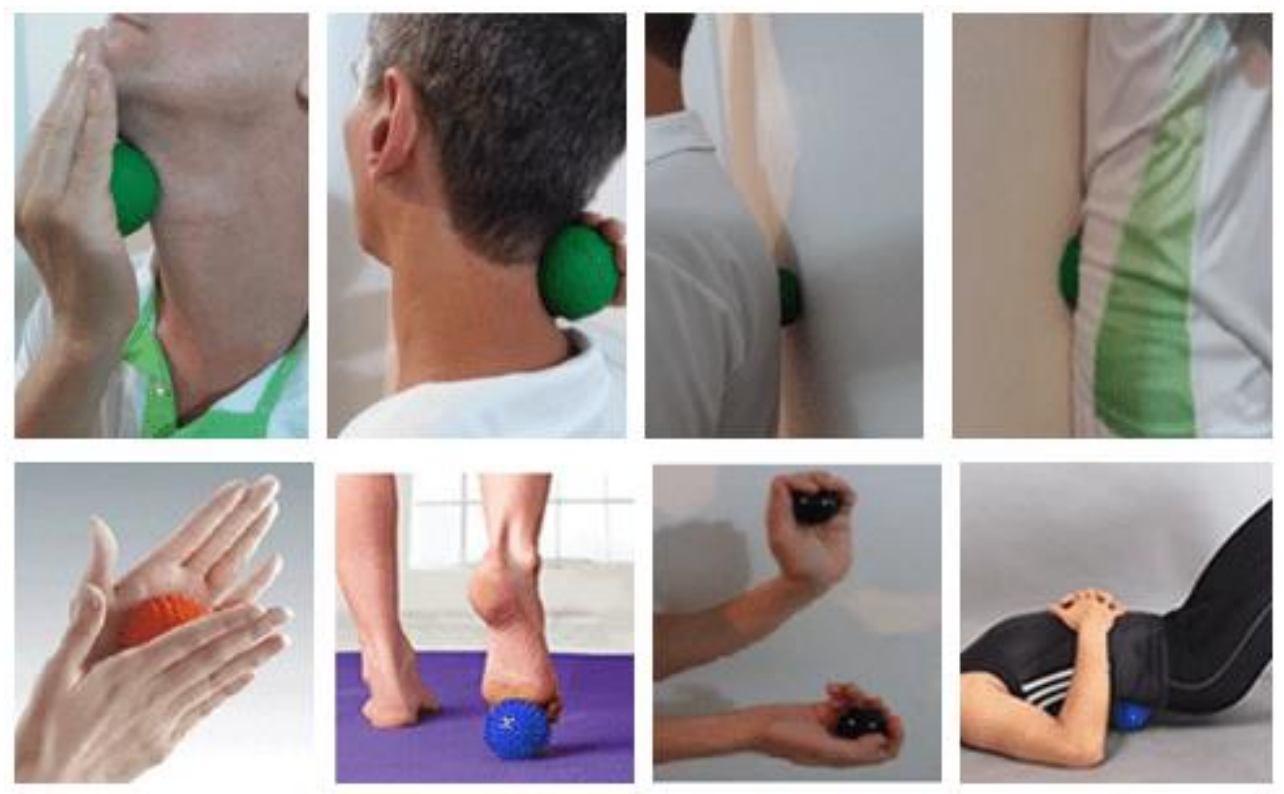

Fonte: Os autores

\subsection{MASSAGGIO PALLA: BENEFICI}

II massaggio a sfera (massaggio a sfera) viene applicato ai tessuti molli ed esercita tre effetti fondamentali: meccanici, fisiologici e psicologici. Gli effetti principali del massaggio sono meccanici, ma generano effetti fisiologici e psicologici.

Il massaggio è una tecnica che promuove la manipolazione sui tessuti molli e come qualsiasi tipo di massaggio rilassante, permette la diminuzione delle tensioni muscolari, oltre a stimolare la produzione di serotonina e istamina che hanno l'azione vasodilator, aumentando sangue e linfatico. II massaggio può attivare i rmorecettori e aumentare la permeabilità della membrana cellulare, questo permette l'assorbimento 
dei nutrienti e conseguente migliore nutrizione cellulare, ossigenazione e idratazione (CLAY, 2008).

Psicologicamente, massaggio palla è in grado di fornire il rilassamento muscolare e mentale, causando uno stato incline a dormire. È noto che il massaggio stimola il rilascio di ormoni endorfine e serotonina che sono responsabili della sensazione di piacere. (PEREIRA, 2013). Ormoni endorfine e serotonina consentono il rilassamento e allo stesso tempo sono quelli responsabili del sonno profondo, che è designato con sonno REM. Inoltre, il massaggio riduce il cortisolo che è uno dei responsabili dell'insonnia /stress, quindi una persona che riceve il massaggio avrà meno cortisolo nel flusso sanguigno, che causerà un miglioramento della loro qualità del sonno $\mathrm{e}$ diminuendo l'ansia e lo stress.

I movimenti di allungamento, compressione, trazione e attrito che vengono eseguiti nel massaggio a sfera esercitano evidenti forze meccaniche sui tessuti. Queste forze meccaniche esercitano effetti di mobilitazione di ammorbidimento e stretching sulla pelle, tessuto sottocutaneo, viscere e muscoli.

L'effetto meccanico si riferisce alle influenze dirette che il massaggio gioca sui tessuti molli massaggiati (CASSAR, 2001). Fornisce effetti fisiologici sul muscolo, nervoso, sistemi circolatori e digestivi. Massaggio con palle quando lentamente la rimozione dei muscoli stimola il riflesso tendineo che riduce la tensione muscolare e si traduce in rilassamento muscolare. Con il rilassamento muscolare scheletrico e le manovre scorrevoli fatte con le palle è possibile esercitare una pressione sufficiente per sposto i liquidi nella direzione del flusso circolatorio venoso o linfatico. Se le manovre sono vigorose, possono provocare aumenti significativi del flusso sanguigno a causa di fenomeni di vasodilazione e capillarizzazione. Questi effetti circolatori guidano gli scambi tra i mezzi cellulari e del sangue, migliorano l'ossigeno e l'input nutrizionale, aiutano nell'eliminazione dei residui dal metabolismo e dall'anidride carbonica. Manovre con pressione più sudabile, ritmica e applicata verso i percorsi dei vasi linfatici superficiali permettono di accelerare il flusso di ritorno linfatico, essendo utili nei casi di edema per aiutare nel riassorbimento. 
I principali effetti meccanici prodotti dal massaggio sono: la mobilitazione del sangue venoso, linfa, lingema ed ematoma, contenuto intestinale, muco, fibre muscolari e massa, tendini, pelle e tessuto sottocutaneo, adere e tessuto cicatriziale (governo statale del Cear, 2010).

Mettiamo anche in evidenza gli effetti fisiologici del massaggio della palla sul sistema digestivo che migliorano il transito intestinale. II massaggio stimola la peristalsi per promuovere l'evacuazione di appartamenti e feci dall'intestino crasso, facilitando la funzione escretoria in caso di costipazione intestinale.

Indubbiamente la riduzione del dolore quando si applica il massaggio si ottiene con diversi meccanismi, tra la diminuzione dello spasmo muscolare e dell'edema. Pertanto, il miglioramento della circolazione di ritorno assorbe l'edema e con esso i metabolici metabolici cellulari. Si riduce anche attivando i recettori della pelle che inibiscono il dolore dal meccanismo del cancello (STARKEY, 2017).

Il rilassamento muscolare e il miglioramento della circolazione contribuiscono già a diminuire le algie muscolo-scheletriche. Tuttavia, il massaggio a sfera riduce il dolore perché obbedisce ai fenomeni della teoria delle inondazioni. Questa teoria ci permette di capire che ci sono diversi tipi di fibre nervose e ognuno ha una velocità di condurre impulso nervoso e una funzione. In questo caso, il dolore fibra nervosa è più lento di tatto e pressione fibra nervosa. Pertanto, quando una persona sente un dolore muscolare e viene massaggiata, gli impulsi nervosi di tocco e pressione arrivano prima nel midollo spinale e inibiranno le vie nervose del dolore che porterebbero informazioni sul dolore al talamo (sito del cervello che permette di sentire quella del r).

L'effetto del massaggio sul controllo del dolore è così importante che viene utilizzato come cura palliativa in pazienti con cancro allo stadio avanzato. Un fatto che si trova nella dichiarazione di florentine (2012, n.p.) che riporta che "il massaggio è una tecnica utilizzata come terapia complementare nei pazienti oncologici, con l'obiettivo di fornire sollievo dal dolore." 
Va sottolineato che per gli effetti inibitori accada, è necessario che le manovre di massaggio con le palle siano fatte costantemente, lentamente e senza intoppi, in modo che l'alloggio si verifichi aumentando la soglia di percezione del nuovo stimolo.

II massaggio a sfera aiuta nel rinnovamento delle cellule epidermiche e consente alle ghiandole sebacee di essere chiare e lavorare meglio. E a causa dei fenomeni vasodilatori e capillarizzazione, la pelle diventa più nutrita e ossigenata, oltre a facilitare la permeazione di alcuni beni cosmetici.

La massaggioterapia può esercitare un'influenza sul sistema immunitario, in quanto agisce sugli strati della pelle che risponde positivamente. (CARVALHO e ALMEIDA, 2018).

\subsection{MASSAGE PALLA: ORIENTATIONS, INDICATION E CONTRAINDICATION}

Prima di applicare il massaggio con le palle è necessario che il terapeuta faccia una valutazione (anamnesi) del cliente. La valutazione fornirà informazioni utili per scegliere se la persona da massaggiare e permetterà di selezionare le manovre prioritarie da applicare. Durante la valutazione, se il cliente presenta una controindicazione relativa, deve essere istruito a consultare un altro professionista della salute e può essere massaggiato solo se ha un'autorizzazione scritta da parte del medico o del fisioterapista.

I principali dati che dovrebbero essere presenti nel modulo di valutazione sono: dati di identificazione (nome completo, indirizzo, numero di telefono, data di nascita, stato civile e professione), dati sullo stato di salute (se la persona è sana o ha qualsiasi malattia: diabete, ipertensione o ipotensione, malattie cardiache, cancro, emicrania, epilessia, costipazione, dismenorfa, cistite, allergie, insonnia, depressione, ecc.) ed è necessario sapere se la persona è incinta o all'allattamento al seno. Altre informazioni che possono contenere nella forma di valutazione riguardano la pratica dell'esercizio fisico, l'alimentazione, il consumo di bevande alcoliche, tabacco e farmaci in uso. 
L'applicazione della massoterapia a sfera presta attenzione alle stesse controindicazioni di qualsiasi applicazione di massaggio e possono essere assolute, cioè il massaggio non può essere applicato al cliente; o relativo, cioè, è possibile applicare il massaggio, ma alcune cure dovrebbero essere adottate o alcune aree non possono essere massaggiate.

Le principali controindicazioni assolute sono trombosi o danni vascolari instabili, stato febbrile, presenza di infezione, cancrena, malattia renale, malattia cardiaca avanzata, forte mal di testa, cambiamenti incontrollati della pressione sanguigna da farmaci, Intossicazione. Le controindicazioni relative sono: fratture (prima solidificate), ustioni recenti, ferite aperte. (VERSAGI, 2015). Altre controindicazioni relative sono: cancro, malattie mentali, decalcenziazioni e gravidanza.

Le principali indicazioni sono: sollievo dallo stress, costipazione, emicrania, recupero della persona in caso di lesioni traumatiche (distorsione, lividi) dopo il rilascio medico, ridurre le tensioni muscolari e tendinose, mialgia per sforzo, prevenzione di STD, drenaggio di edema venoso e linfatico, adiuvante nel trattamento delle smagliature, lipodistrofia localizzata, paniculopatia fibrosclerotica eedema, insonnia, ansia, ecc.

\subsection{MASSAGGIO PALLA: TECNICHE}

Alcune regole di base per l'applicazione del massaggio dovrebbero essere seguite, come: sia il massaggiatore che il cliente dovrebbero sentirsi a proprio agio durante la sessione; contatto con il cliente non deve essere improvvisamente rimosso; il massaggio deve essere interrotto nelle regioni in cui il paziente sente dolore; non premere o massaggiare direttamente sulla colonna vertebrale. (ELLSWORTH, 2012).

Le manovre di massaggio a sfera devono rispettare i principi di direzione, pressione, velocità, ritmo e durata in base agli obiettivi desiderati.

La direzione dipenderà dall'effetto desiderato, quindi può essere eseguita nella direzione di circolazione che si desidera aumentare o nella direzione delle fibre muscolari da massaggiare. Per quanto riguarda il flusso arterioso, le manovre devono 
essere centrifughe o quasi discrepanti per massaggiare gli arti, il tronco e il collo e centripili o prossimali per massaggiare la testa. Se la direzione del flusso è venosa, le manovre devono essere centripete o questo-prossimal per massaggiare arti, tronco e collo e centrifughe o quasi distale per massaggiare la testa. Se si mira al drenaggio linfatico è necessario seguire la direzione del flusso dei vasi linfatici e mantenere la sequenza di drenaggio proposta da José Maria Pereira de Godoy e Maria de Fatima Guerreiro Godoy.

Per quanto riguarda la pressione da esercitare, dovrebbe essere confortevole e piacevole quando si cerca il rilassamento muscolare e la riduzione del dolore. È interessante notare che è necessario che $\|$ feedback dei clienti per regolare la pressione. Ci sono persone più muscolose 0 obese dove la pressione tenderà ad essere leggermente superiore rispetto a magra, anziani o bambini. Se si mira al drenaggio linfatico, la pressione dovrebbe essere compresa tra 30 e $40 \mathrm{mmHg}$.

Anche la velocità e il ritmo variano a seconda delle esigenze. Le manovre di rilassamento e drenaggio devono essere senza rallentamenti, costanti e ben ritmici. Per la dinamizzazione circolatoria arteriosa, il trattamento di striatura, la lipodistrofia localizzata, il fibroedemageloid dovrebbe essere più veloce. È bene ricordare che $\mathrm{i}$ movimenti eseguiti lentamente tendono ad essere rilassanti, mentre il contrario, più stimolante.

II massaggio dura da 5 a 20 minuti quando applicato in una sola regione, tranne se I'obiettivo è il drenaggio linfatico, in questo caso durerà da 30 a 40 min. Quando applicato in modo sistemico, cioè in tutto il corpo, mirando al rilassamento muscolare e alla diminuzione delle tensioni emotive, durerà da 45 a 90 min.

Va notato che il tempo dipenderà fortemente dall'obiettivo da raggiungere, e dalle dimensioni della zona del corpo, dal momento che ci vuole meno tempo per trattare una persona relativamente piccola rispetto a una persona grande. Nelle persone molto giovani e molto anziane, la durata del massaggio dovrebbe essere ridotta, perché l'arco riflesso è più sensibile e l'effetto integrale si ottiene più velocemente. 
II massaggio a sfera applicato dal terapeuta è diviso in quattro manovre di base e ogni manovra ha le sue variazioni. La manovra che avvia e termina il servizio è quella di scorrimento, che consiste nello scorrimento su o con le palle sulla superficie del corpo del cliente sempre in risposta al flusso circolatorio desiderato o alla direzione delle fibre muscolari o al senso di peristalsi (Figura da 4 a 11).

Figura 4: Tecnica simmetrica di slip-through longitudinale in Mmli.

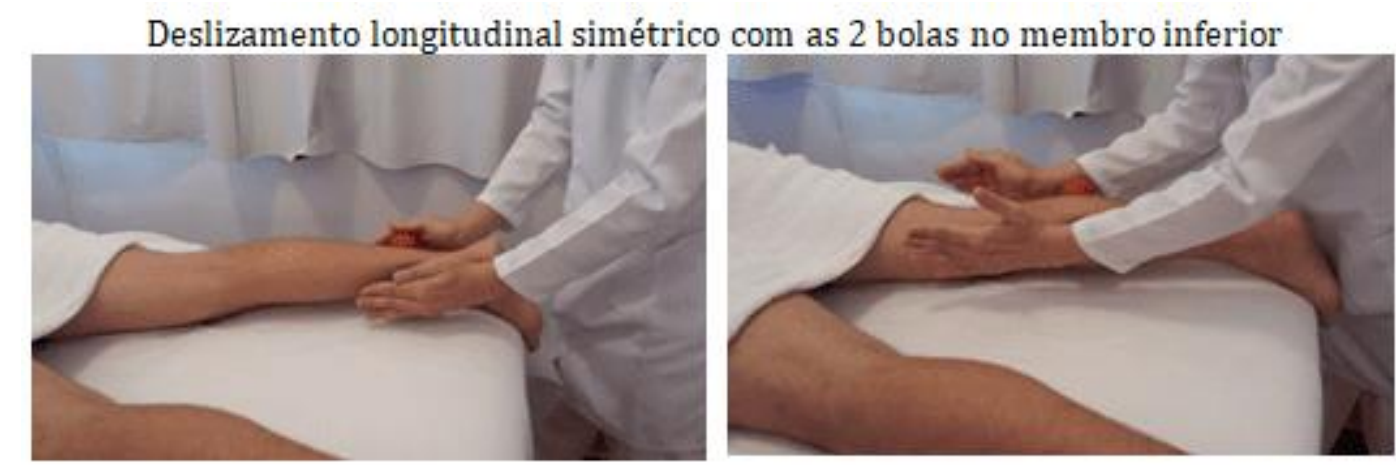

Fonte: Os autores

Figura 5: Tecnica asimmetrica di slip-up longitudinale nel bagagliaio.

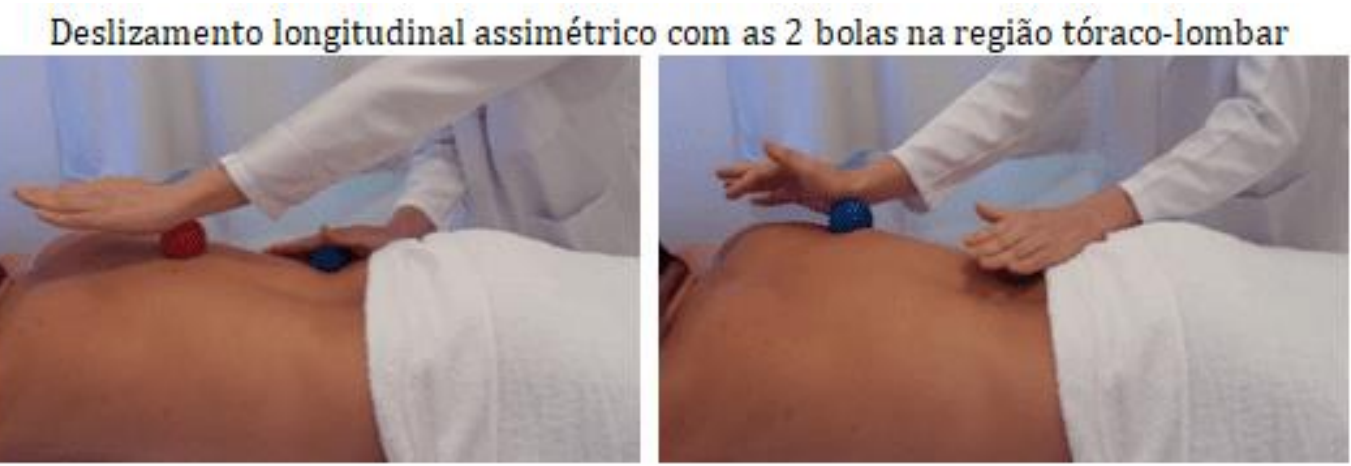

Fonte: Os autores 
Figura 6: Tecnica cross-slip.

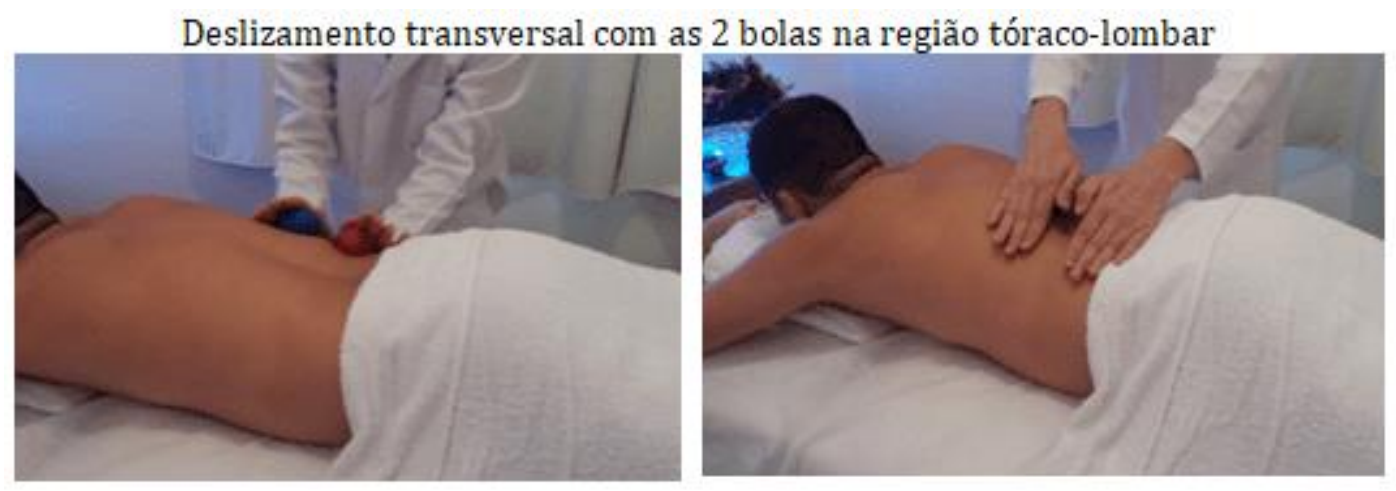

Fonte: Os autores

Figura 7: Tecnica simmetrica di slip-up longitudinale nel bagagliaio.

Deslizamento longitudinal simétrico com as 2 bolas na região tóraco-lombar
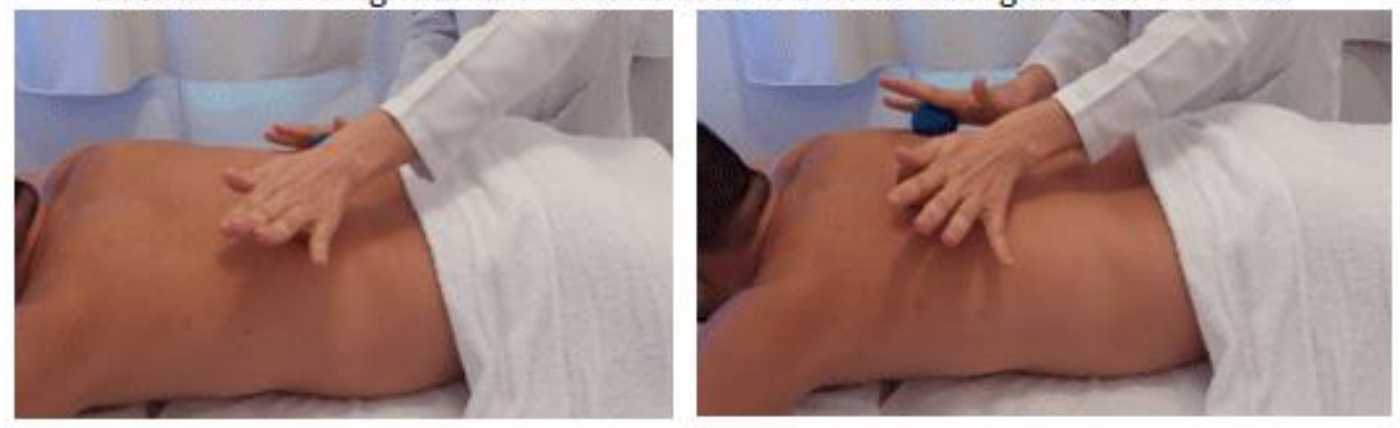

Fonte: Os autores

Figura 8: Tecnica di scorrimento circolare.

Deslizamento circular com uma bola na região abdominal no sentido do peristaltismo. A manobra pode ser executada com uma ou duas mãos.

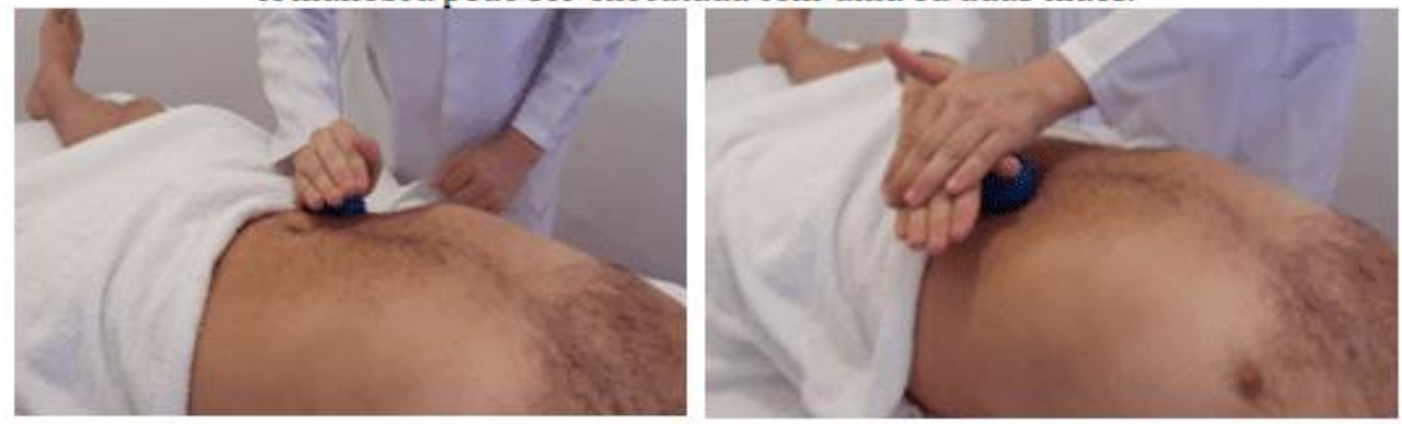

Fonte: Os autores

RC: 41888

Disponível em: https://www.nucleodoconhecimento.com.br/salute/massoterapia-con-palle 
Figura 9: Tecnica simmetrica di slip-up longitudinale sull'avambraccio.

Deslizamento longitudinal simétrico com as 2 bolas na mão e no antebraço
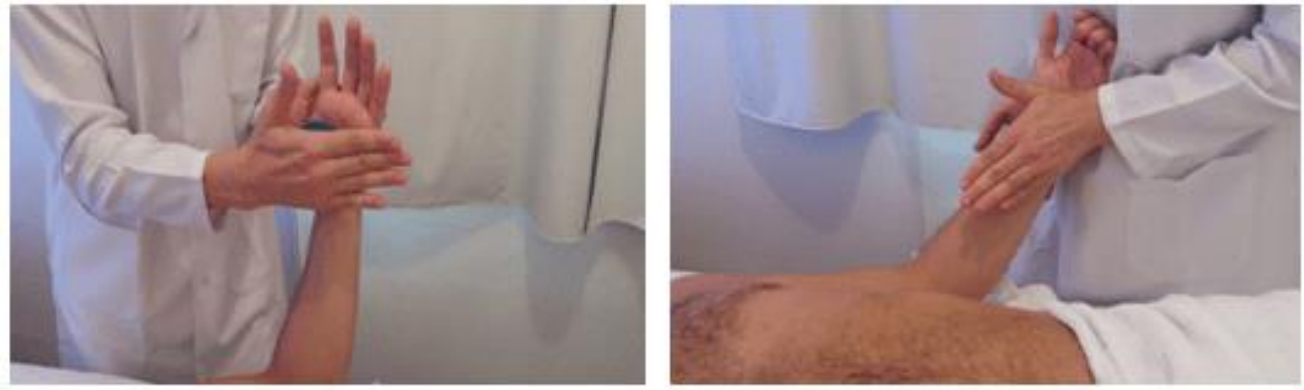

Fonte: Os autores

Figura 10: Tecnica simmetrica di slittamento longitudinale sul braccio.

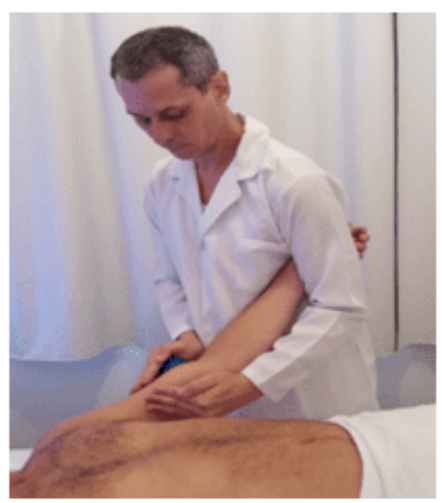

Deslizamento longitudinal simétrico com as 2 bolas no braço. Observe que o terapeuta sustenta o membro superior fixando-o entre o tronco e o braço. É fundamental que o cliente não sustente 0 membro.

Fonte: Os autores

Figura 11: Tecnica di scorrimento diagonale.

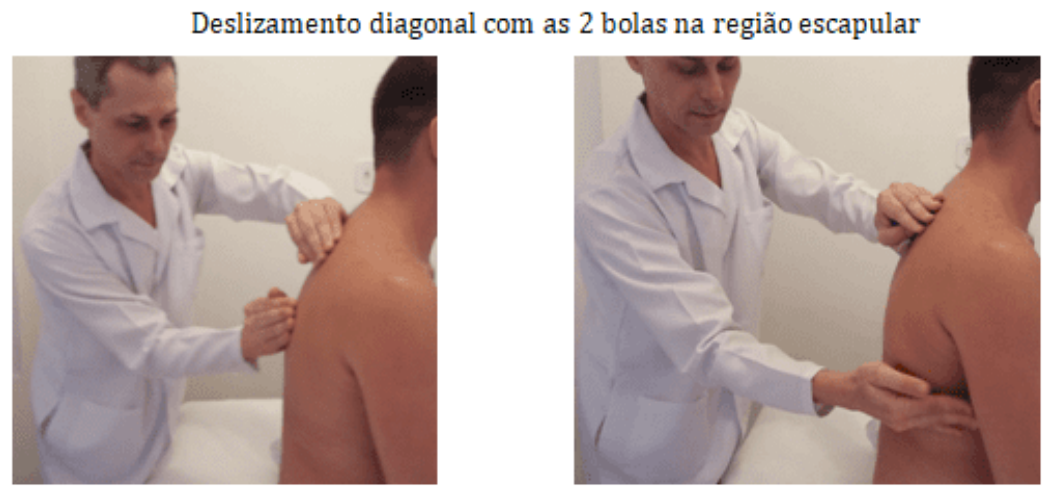

Fonte: Os autores

RC: 41888

Disponível em: https://www.nucleodoconhecimento.com.br/salute/massoterapia-con-palle 
La seconda manovra è la compressione fissa o la compressione e trascinare. II primo è quello di mantenere la regione compressa dalla palla per 10 a 15 secondi o per tutta la sessione. II secondo, consiste nel comprimere la palla contro il corpo e trascinarla in movimenti circolari (Figura 12 e 13).

Figura 12: Tecnica di compressione nel piede.
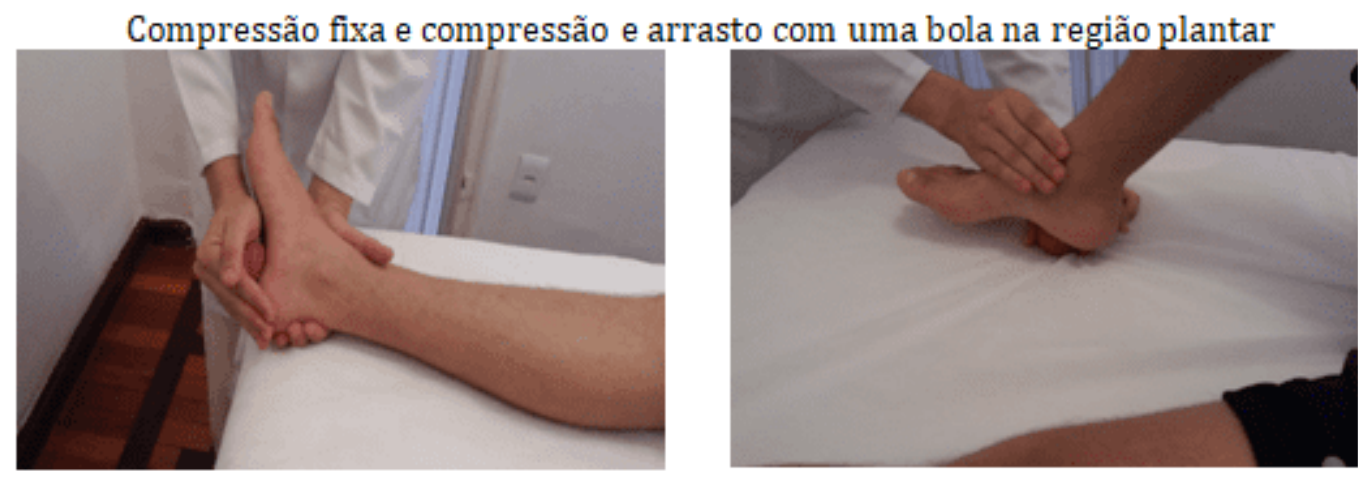

Fonte: Os autores

Figura 13: Tecnica di compressione nella colonna vertebrale.

Compressão fixa com as 2 bolas na região paravertebral cervical e lombar
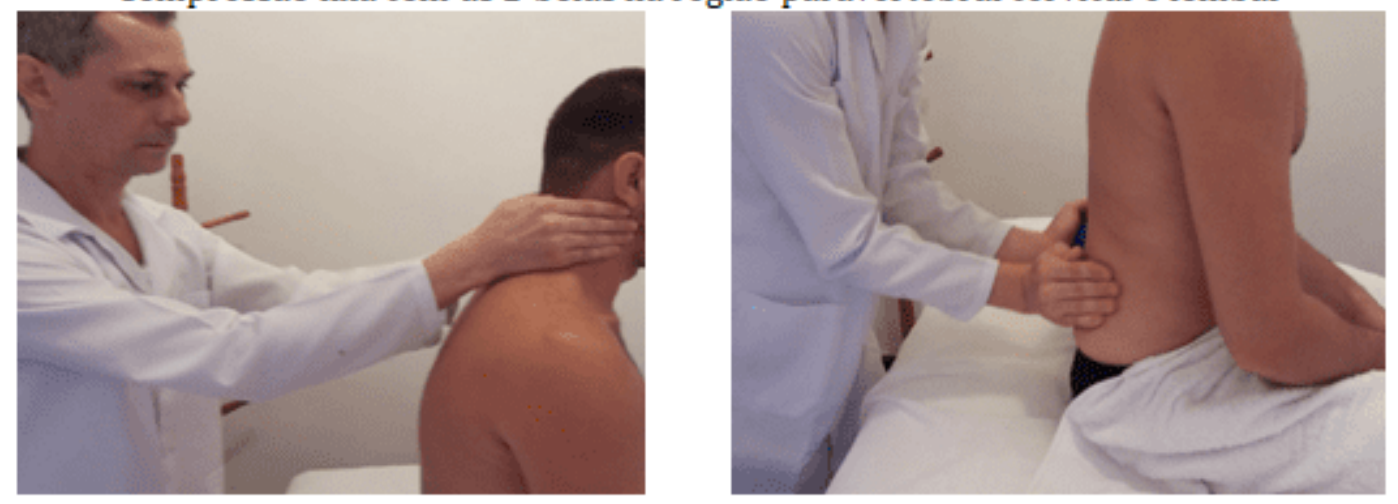

Fonte: Os autores

La terza manovra è impastare e in questo caso vengono sempre utilizzate due palle. II terapeuta impasta il tessuto molle tra le palle con una buona pressione o forte pressione, ma non scomodo. II cliente non può segnalare dolore o fastidio in qualsiasi ipotesi (Figura 14). 
Figura 14: Tecnica di impastamento.

Amassamento de trapézio e da loja posterior de perna
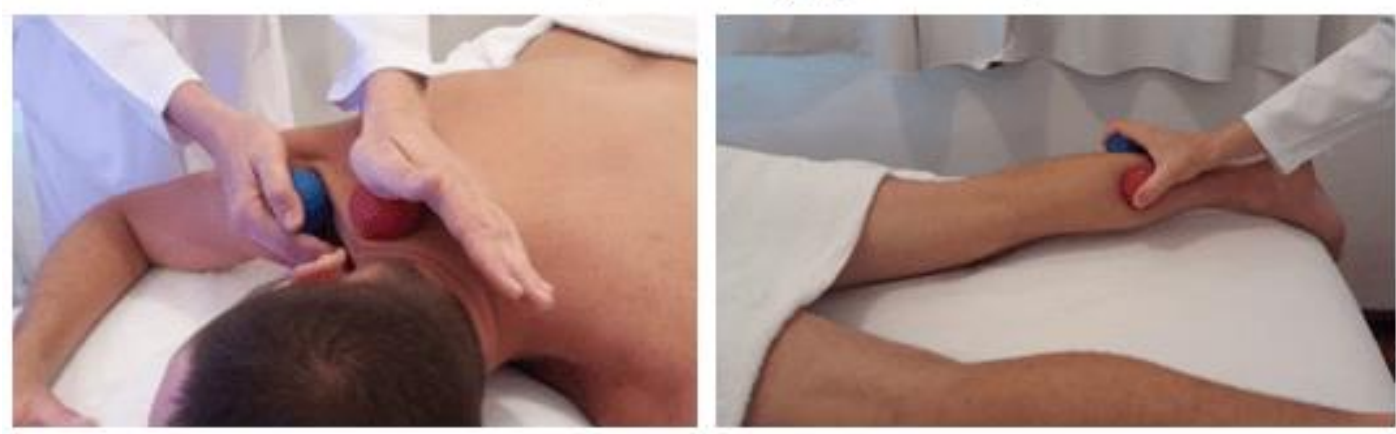

Fonte: Os autores

La quarta manovra di base è l'attrito e in questo caso, di solito viene utilizzato in piccole regioni e con una sola palla. Questa manovra è una sorta di slittamento profondo, eseguito vigorosamente e rapidamente, al fine di ridurre i noduli di tensione muscolare, annullare i coaguli, aumentare la microcircolazione locale. Provoca un grande riscaldamento della regione massaggiata ed è raccomandato in caso di striastrinie e lipodistrofia localizzata. Le manovre mirano a riscaldare la regione e sono fatte con la palla che circola tra il palmo e la superficie del corpo del cliente (Figura 15).

Figura 15: Tecnica di attrito.

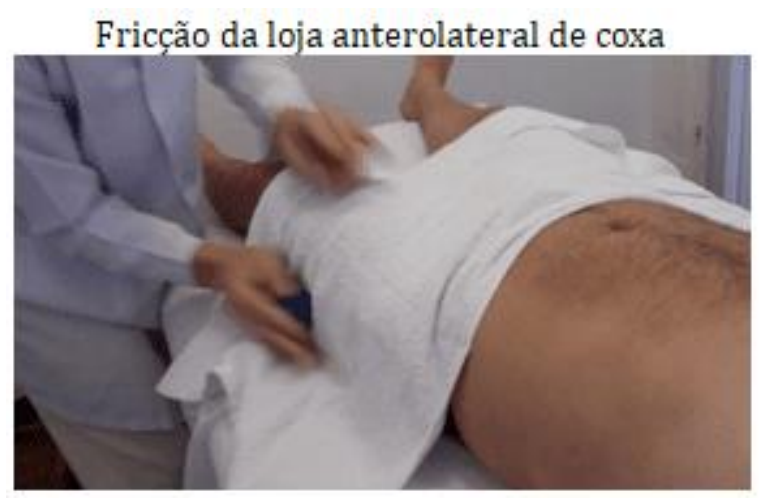

Fonte: Os autores

Ci sono variazioni di ciascuna delle manovre di base, oltre alla tecnica di Auto Massaggio con palle, che la persona applica il massaggio su di esso (Figura 16). 
Figura 16: Tecnica di auto-massaggio.

\section{Self Ball Massage}

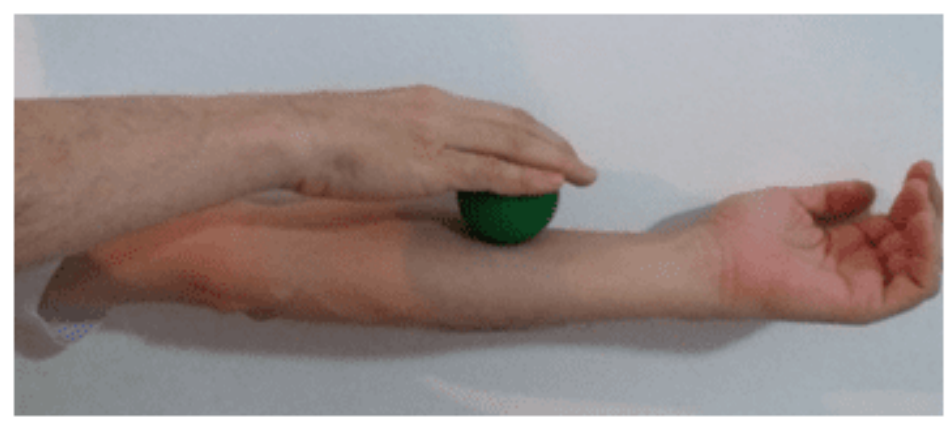

Fonte: Os autores

\section{CONCLUSIONE}

Le prime testimonianze storiche della massoterapia risalgono al 1800 a.C. e si trova nei libri Ayur Veda, ma è noto che gli egiziani hanno usato il massaggio per scopi terapeutici per più di 4000 a. C. E fu nella dinastia Ming (1368-1644 d.C.) che un artigiano delle armi creò due sfere di ferro basate sul concetto di yin/yang. Hanno cominciato ad essere impiegati per fornire effetti terapeutici e per migliorare la condizione fisica e allenare il temperamento (diminuire l'ansia, calmarsi).

Al giorno d'oggi le palle di ferro sono ancora utilizzati, ma altri materiali emersi con legno, silicone, gomma e molti altri. Tutte queste palle, indipendentemente dal materiale, servono a massaggiare ed esercitare e possono fornire benefici organici e psicologici già riconosciuti nella minoterapia. Si può vedere che il massaggio palla applicato dal terapeuta è diviso in 4 manovre di base, tuttavia ogni manovra ha le sue variazioni.

\section{RIFERIMENTI}

BERTOJA, Vanessa Gomes Bertoja; TOKARS, Eunice. Os benefícios da massagem relaxante.

em:

https://www.librarybus.com/view?t=os+benef\%C3\%ADcios+da+massagem+relaxant e+-+TCC+On-line+- 
+Tuiuti\&u=http\%3A\%2F\%2Ftcconline.utp.br\%2Fmedia\%2Ftcc\%2F2017\%2F05\%2F OS-BENEFICIOS-DA-MASSAGEM-RELAXANTE.pdf. Acesso em: 10 jan.2018.

BRAUN, Mary Beth; SIMONSON, Stephanie. Massoterapia. São Paulo: Manole, 2007.

CANNECCHIA, Marcela Cleto. Benefícios da massagem relaxante para o homem da atualidade. Pesquisa e Ação. Mogi das Cruzes, SP. v.5, n.1, p. 46-49. Jun. 2019. Disponível em: https://revistas.brazcubas.br/index.php/pesquisa/article/view/574/703. Acesso em: 05 ago. 2019.

CARVALHO, Rosemeire de Jesus; ALMEIDA, Maria Antonieta Pereira Tigre. Efeitos da Massoterapia sobre o Sistema Imunológico. Id on Line Rev. Mult. Psic. v.12, n. 40. p.353-366, 2018

CASSAR, Mario-Paul. Manual de Massagem Terapêutica: um guia completo de massoterapia para o estudante e para o terapeuta. São Paulo: Manole, 2001.

CLAY, James. H.; POUNDS, David. M. Massoterapia Clínica: integrando anatomia e tratamento. São Paulo: Manole, 2008.

ELLSWORTH, Abigail. Massagem: anatomia ilustrada, guia completo de técnicas básicas de massagem. Barueri, São Paulo: Manole, 2012.

Florentino, Danielle de M. A fisioterapia no alívio da dor: uma visão reabilitadora em cuidados paliativos. Brazilian Journal of Health and Biomedical Sciences. Rio de Janeiro; Revista do Hospital Universitário Pedro Ernesto. v.11, n.2, abr/jun, 2012.

FRITZ, Sandy. Fundamentos da Massagem Terapêutica. 2.ed. São Paulo: Manole, 2002.

GOVERNO DO ESTADO DO CEARÁ. Técnico em Massoterapia: técnicas clássicas e modernas de massoterapia. Escola Estadual de Educação Profissional - Ensino Médio Integrado à Educação Profissional - Curso Técnico em Massoterapia. Fortaleza Ceará, 2010. 
PEREIRA, Maria de Fátima Lima. Recursos Técnicos em Estética. Vol. I. Série Curso de Estética. São Paulo. Difusão Editora. 2013.

RAMOS, Andréa Rosa. Técnicas de massagem corporal. Londrina: Editora e Distribuidora Educacional S.A, 2017

SILVA, Carina Geremias dos Santos. Massoterapia Mãos de Anjo. Plano de Negócios. PG, Gravataí, v. 2, n. 2, 2016. Disponível em: http://revista.faqi.edu.br/index.php/PN/article/view/259/277. Acesso em: 02 ago 2019.

STRARKEY, Chad. Recursos Terapêuticos em Fisioterapia. 4 ed. São Paulo: Manole, 2017.

VERSAGI, Charlotte Michael. Protocolos Terapêuticos de Massoterapia: técnicas passo a passo para diversas condições clínicas. Barueri, São Paulo: Manole, 2012.

WOOD, E. C; BECKER, P. D. Técnicas de Massagem de Beard. 5. ed. Rio de Janeiro: Elsevier, 2008.

Inviato: agosto 2019.

Approvato: ottobre 2019. 